\title{
Los Fundamentos Teóricos del Derecho Inglés
}

\author{
Por el Doctor ROBERTO MAC LEAN *
}

La corriente filosófica que más influencia ha ejercido en Inglaterra durante los últimos treinta años, puede ser resumida diciendo que la Teoría del Derecho es ante todo definición de palabras (1). Esta tendencia que presta especial atención al uspecto semático es conocida con el nombre de Filosolía Lingüística o Escuela Analítica de Cambridge, y se remonta en sus antece. dentes más distantes a la teoría del significado de las palabras de John Stuart Mill. El interés despertado por los uilitaristas en el uso del lenguaje. así como sus exigencias de precisión, atemperada por el sentido común y por el entendimiento de que no se trata de evitar el uso de metáforas sino de no dejarse llevar por ellas, constituye un componente importante del legado intelectual inglés (2). Los utilitaristas sólo plantearon aquellos problemas que filósofos que les sucedieron tales como W. S. Jevons, F. H. Bradley, W. James y, a comienzos de este siglo, G. E. Moore y Bertrand Russeil comenzaron a responder (3). Este último señaló, ya, algunas inconsistencias en la teoría tradicional del significado; pero fue Ludwig Wittgenstein, quien modificó el concepto tradicional al afirmar que el significado de una palabra debe buscarse en su uso en el idioma. Esta filosofía encontró pronta aceptación entre los juristas.

El concepto tradicional consideraba la acción de significar una pala. br $x$, como equivalente a la acción de nombrar o denotar aquello que representa. Este concepto, que da magnificos resultados en el caso de nombres

* Caledrático de la Facultad de Derecho y Ciencias Políticas de la Pontificia Universidad Católica.

(1) Glanvile WILLIAMS. International Law and the Controversy Concerning the Word Law: en, Philosophy, Politics and Society. London, 1956 p. 134. T.D. WELDON. The Vocabulary of Politics. London, 1955. p. 17.

(2) M. J. P. PLAMENATZ. The Legacy of Philosophical Radicalism; en, Law and Opinion in Englund in the 20th Century. London, 1959. p. 27.

(3) Sir Ernest BAKER. Political Thought in England, 1848 to 1914. London, 1954. Giibert RYLE. The Theory of Meaning; en. British Philosophy in the Mid-Century. A Cambridge Symposium. London, 1957. p. 239. Gilbert RYLE and others. The Revolution in Pil sophy. London, 1956 . 
propios gue denotan sujetos fócilmente identificables, resulta inadecuado en el caso de palabras que designan clases de cosas, cosas inexistentes, o nombres abstractos. A comienzos de siglo, Bertrand Russell dió el nombre de "símbolos incompletos" o "expresiones" $\alpha$ estas palabras, cuyo significado no se podía explicar satisfactoriamente con el método tradicional, a la vez que afirmó que saber io que significa una expresión es saber qué cosa es lo que puede decirse, lógicamente, con ella y qué cosa no. Quedó asi en claro que lo contrario de lo que se puede decir verdadera o falsamente, no es lo que no existe, sino lo sin sentido (4). La mayoria de las palabras, por otre lado, no son siquiera sustantivos, sino adjetivos, verbos, conjunciones o preposiciones, que no pueden denotar, significar o ser simbolos de aigo en la misma forma que los nombres propios denoian o son símbolos del sujeto. Esta fue la causa por la que Wittgenstein, y discípulos suyos como H. B. Acton, A. J. Ayer, A. M. Mc Iver y J. A. T. Wisdom, entre otros, desconocieron la doctrina tradicional, salvo en relación con los norabres propios que siempre son atribuídos arbitrariamente, a diferencia de las otras palabras que pretenden describir hechos o relaciones. Sostienen estos filóscíos que para que una oración diga algo falso o verdadero el sujeto debe, ser nenecesariamenie significante; es decir, que no se puede afirmar que una cosa no existe, a menos que antes tenga cierto tipo de existencia para nosotros. una existencia semántica, como símbolo; y esto se puede aplicar a nombres abstractos como "justicia", "certeza", "seguridai" u "homicidio". Witgenstein sostuvo la idea, fundarnental, que saber lo que significa una expresión? consiste en conocer un conjunio de prohibiciones, permisos y obligaciones $o$, en otras palabras, sus diversas acepciones, su uso en el idioma. Una de las metáloras favoritas de este filósofo exphica que el "significado" de un alfil en el juego de ajedrez, sería las formas y direcciones en que puede moverse, así como las jugadas más comunes que un jugador experto puede esperar que se realicen. Conocer, pues, el significado de una expresión equivale a conocer cómo puede ser empleada, cómo no, y las normas que regulan su empleo, que pueden ser las mismas aún para expresiones de diversa composición físia como "Law" Y "Derecho". Una vez que se ha descubierto el uso de las palabras en el idioma, se puede proceder a examinar si lo que describen es verdadero o falso y si, además lo hacen adecuadarnente.

Otra tendencia importante en este siglo, ha sido el desarrollo en el pensamienio jurídico de una moyor conciencia de responsabilidad social (5). Las primeras manifestaciones de este pensamiento se encuentran en Thomos Hobbes, y luego en Teremy Bentham quien sostuvo que el fin último del Dere-

(4) Dennis LLOYD. Introduction to Jurisprudence. London, 1959. p. 32 G. RYLE Op. cit. p. 250. Stuart HAMPSHIRE. The Interpretation of Language: Words and Concepts; en British Philosophy in the Mid-Century. cit p. 267 G.J. WARNOCK. English Philosophy Since 1900. p. 62.

(5) Morris GINSGERG The Growth of Social Responsability: en, Law and Opinion in England in the 20ih Century. London, 1959. p. 3. 
cho es lograr la mayor felicidad del mayor número. Pero fue en realidad debido a los filósofos idealistas de Oxford, y a las enseñanzas de T. H. Green sobre los principios de la obligación política, en particular, que comenzó $\alpha$ producirse un desplazamiento en la opinión pública del individualismo liberal que ciefendía el "laisser faire", hacia el colectivismo que tiende a la utilidad social; desplazamiento que se reflejó más iarde en la legislación. $\bar{A}$. V. Dicey. fue el primero en llamar la otención sobre este fenómeno (6), y aunque algunas de sus predicciones incurrieron en la exageración, especialmente en lo relativo a la desaparición del individualismo, su estudio sobre el Derecho y la Opinión Pública contiene observaciones válidas y establece entre estos elementos una relación que sirvió de base a la Sociología Jurídica en Inglaterra continuada más tarde por Morris Ginsberg y W. Friedman. De esta tendencia, osimismo, se derivó la aceptación de la clasificación del Derecho como una ciencia social y normativa - a la que los ingleses siempre se habían mostracio reticentes - y también significó el fin del aislamiento que el Derecho había guarajado con relación a las demós ciencias sociales (7).

A] examinar el uso de la expresión Fuentes de Derecho, se encuentra en Inglaterra das puntos de vista opuestos: uno represeniado, principalmente, por Hobbes y Austin, y el otro por Locke, Moine y Dicey (8). Para los primeros, el Derecho es impuesto a la sociedad por una voluntad soberana, es arifficial, y crece - por decirlo esí - de arriba para abajo. Para los seguncios el Derecho crece de abajo para arriba, se desarrolla por vitalidad propia y es producto espontáneo, aunque su ejecución y sanción quedan a cargo de una autoridad suprema establecida. Thomas Hobbes fue el prinzipal expositor de la teoría de la soberania del Ezlado (9), que sostiene que la ley es una norma de conducta, impuesta y sancionada por el sobercno. Desde sus primeros libros defendió la teoría que declaraba que el rey tenía poderes absolutos, si bien afirmaba que estos no nacian del mandato divino sino del acuerdo de los hombres para evitar desiruirse. En el "Leviatán" reafirmó su doctrina $y$ dedujo que si no hay soberano na puede haber Derecho; que el Derecho representa la voluntad del soberano, quien ademós es el único llamado a interpretarlo; que no es la sabiduría, sino la autoridad, la que crea el Derecho; y que es el rey el juez supremo y único legislador. Este absolulismo se explica al tomar en cuenta al panorama histórico que le sirvió de fondo, constituído por los disturbios puritanos y por el gobierno de Cromwell. Su cicctrina, sumida en el olvido durante dos siglos, fué puesta nuevamente en circulación en el siglo XIX por John Austin (10), para quien el derecho positivo, propiamente dicho, está conformado, úni-

(6) A. V. DICEY. Law and Public Opinion in England in the 19th Century. 2nd Edition. Lendon. 1914,

(7) Huntington CAIRNS. Low and the Socia! Sciences. Loncion, 1935. p. 1 y siguientes.

(8) Sir Carleton K. ALLEN. Low in the Making. Oxtord, 1961. p. 1 .

(9) G. P. GOOCH. Political Thought in England. From Bacon to Halifax. Oxlord, 1950. p. 23.

(10) Sir Paul VINOGRADOFT. Common Sense in Law. Oxford, 1959, p. 24. Williams L. DAVIDSON. Folitical Thought in England. The Utilitarians. Oxford, p. 165. 
camente, por la ley impuesta, como orden o mandato, por seres políticamente superiores a seres políticamente inferiores. Si bien este autor reconoce $y$ acepta la existencia de diversas fuentes de Derecho mediatas a inmediataz, directas, indirectas y oblicuas, insiste, repetidamente, que el término fuente corresponde, con propiedad, sólo al autor directo o inmediato de la ley, es decir, al soberano cuya naturaleza es inalienable, indivisible e imprescriptible; la única división de poderes que acepta es entre poder supremo y woderes subordinados (11). Durante muchos años Austin fue considerado como el jurista de mayor influencia no sólo en Inglaterra, sino en todos los paises que se rigen por el sistema legal anglosajón; pero las recientes investigaciones históricas y antropológicas han desautorizado mucho de los fundamentos de su doctriner inspirados en Hobbes. El instinto de agruparase no nuce en los hombres, como decían Hobbes y Austin, del temor de destruirse a si mismos, sino de la cooperación para satisfacer necesidades comures. $Y$ cualquiera que sea el instrumento constitucional que asegure la observancia y cumplimiento de la ley, no hay ninguna justificación histórica para afirmar que es necesario que sea un ser políticamente superior.

El segundo punto de vista, que sostiene que el Derecho se desarrolla por vitalidad propia, se originó en la doctrina de Richard Hooker, sobre política eclesiástica anglicana en el siglo XVI, que sostiene que el verdaderu fundamento del Derecho está en el consentimiento común (12). Más tarde, el filósofo de la Revolución de 1688 John Locke, desarrolló esta iriea y afirmó, contrariamente a lo que defendía Hobbes, que los hombres nacen iguales, y que esta igualdad se deriva del consentimiento común a un contrato social en que el Derecho se expresa por la voluntad de la mayoria. Un gobierno absoluto, afirmaba, no es gobierno, pues se guía por el capricho y no por la razón, y carece del elemento esencial para la obligatoriedad de la ley: el consentimiento (13).

Las críticas más concluyentes a la doctrina de Hobbes y Austin provinieron, no obstante, de Sir Henry Maine que demostró, con ejemplos do civilizaciones antiguas y de pueblos primitivos de Oriente, que lejos de señalar principios universales y permanentes sobre el Derecho y la sociedad, Hobbes y Austin hacían referencia a una sola fase del desarrollo de la civilización occidental, aquella que se había regido por la concepción bizantina de la monarquía. Sir Henry Maine, en su conocida obra "Derecho Antiguo", fue el más destacado representante en Inglaterra de la Escuela Histórica, y defendió la doctrina que el Derecho, como fruto del genio ori. ginal de un pueblo no debe ser reformado sin tener en cuenta el pucblo

(11) John AUSTIN Lectures on Jurisprudence, London, 1885. Lecture XXVIII.

(12) Christcpher MORRIS. Political Thought in England. Tyndale to Hooker. Oxford, 1953. pp. 180 y siguientes.

(13) John LOCKE. An Essay Concerning the True Original Extent and End of Civil Government. Oxford, 1948. Harold J. LASKI. Political Thought in England. Locke to Bentham. Oxford. 1955 p. 19. 
de que se trata (14). La aparición de "El Origen de las Especies" de Darwin aporto nuevos elementos de juicio a favor de este punto de vista: asi como la Escuela Orgánica o Sociológica de Herbert Spencer que inspiró con T.H. Green a A. V. Dicey, en su trabajo sobre la Opinión Pública y el Derecho. De estas observaciones se concluye que no se puede iniciar el estudio de las fuentes del Derecho inglés desde un solo punto de vista, cualquiera que sea este.

La costumbre es la más antigua de las fuentes y aunque su importancia es de primer orden en las sociedades primitivas, su función en el Derecho moderno no debe, ni puede, desestimarse (15). Aun Austin, quien consideraba como Derecho sólo el que emanaba directamente del soberano, no pudo cerrar los ojos a la realidad de la costumbre, a la que dió el nombre de Moral Positiva, sin explicar satisfactoriamente la diferencia intrínseca entre esta y el Derecho. La costumbre como fuente de Derecho nace de la conveniencia que trae su uso a la sociedad y al individuo, es decir de la convicción común, de la conciencia colectiva de su obligatoriedad - a distinción de la costumbre meramente social - y de la cooperación; y aunque es un error común definir el Derecho inglés como consuetudinario (16), ha sido unánimemente reconocido que tuvo su fuente de inspiración en la costumore medioval (17). Esta influencia es más evidente en lo relativo a la propiecad, la familia y algunas instituciones sociales; pero aún en nuestros clías en el Derecho Constitucional tienen vigencia $y$ eficacia normas no promulgadas. A diferencia de la mayor parte de países, Inglaterra carece de una constitución escrita; la estructura legal del Estado está prevista en algunas leyes $y$ en numerosos usos y reglas observados estrictamente, aunque jamás hayan sido estos últimos promulgados o aprobados por autoridail alguna (18). De este modo, conforme a lo establecido por la ley escrita, corresponde a la Reina el nombramiento del Primer Ministro y de los demás miembros del Gabinete, pero en la práctica siempre debe consultar la opinión del jefe del partido en el gobierno. De la misma manera no hay ley escrita que determine la forma de gobierno por gabinete ministerial, ni

(14) Sir Henry Summer MAINE. Ancient Law. Oxford. 1959.

(15) Sir Carleton K. AlLEN. Op. cit. p. 64. Sir Paul VINOGRADOFF Op. cit. p. 107. Dennis LLOYD. Op. cit. p. 331. A. K. R. KIRALFY. The English Legal System, Third Edition. London, 1960. p. 130. O. HOOD PH_LLIPS. A First Book of English Law Third Edition. London, 1955. p. 159. G. R. RUDD. The English. Legal System. London, 1962. F. 21 .

(16) El autor cometió inadvertidamente este error en El Sistema Legal Inglés. Revista de Jurisprudencia Peruana. 1958. $\mathrm{N}^{\circ}$ 179. p. 1286.

(17) En el primer tratado sistemática sobre el Derecho inglés, su autor, Blackstone, lo divide en: a) costumbres generales; b) costumbres particulares; y c) ciertas leyes que por costumbre son aplicadas en ciertos tribunales. Se consideraba, entonces, que la legislación y las decisiones de los tribuncles ercon otros tantos medios de crear costumbres

(18) Fhilip S. JAMES. Introduction to English Law. Third Edition. London, 1955, p. 127. S. B. CHRIMES. English Constitutional History. Oxford, 1949. p. 8. Geoffrey MARSHALL. Some problems of the Constitution. London, 1961. p. 28. 
la estructura y funcionamiento de éste, y jamás han ocurrido problemas que hagan pensar que esto es inconveniente. Pero estas convenciones no son lc que se entiende por costumbre jurídica en sentido estricto. Tampoco son costumbres jurídicas los usos generales de comercio, pues la costumbro debe ser una norma excepcional y limitada, ya sea en su aplicación geográfica o en la clase de personas a quienes se aplica (19), y su sanción obligatoria debe ser completa y uniforme. La costumbre es la expresión del carácter peculiar de cada pueblo, y se forma y propaga debido a la fuerza de imitación, independiente de factores lógicos y utilitarios, si bien el elemento más importante para su consolidación reside en su interpretación y aplicación judiciales. Investigaciones históricas recientes han sugerido que las costumbres medievales en que tuvo su origen el Derecho inglés, no ercn en realidad costumbres del pueblo, sino de los tribunales de justicia. Vinogradoff ha explicado esto, en parte, al afirmar que la costumbro era el Derecho de la época en que los jueces $Y$ el pueblo que representaban mantenían, todavía, un íntimo contacto (20). La interpretación judicial de la costumbre se ha convertido en una técnica especializada, y para que aquella sea reconocida debe cumplir con ciertos requisitos que están destinzajos a probar su existencia y su aplicación reales.

Estos requisitos, una vez probada la existencia de la costumbre, son, primero, que debe haber existido desde tiempo inmemorial. Esta expresión describe, en el Derecho inglés, un período de tiempo mucho más preciso de lo que parece indicar a primera vista; concretamente, desde antes del año 1189 (21). La carga de la prueba recae sobre la persona que cita la costumbre en su apoyo. No obstante, si una costumbre ha existido durante largo tiempo se presume, salvo prueba en contrario, que existía en 1189. El segundo requisito es la continuidad de la costumbre desde que fué establecida; y se hace una distinción entre la continuidad del uso del derecho que crea la costumbre - que si puede ser interrumpido - y la del derecho mismo que debe ser continuo. El tercer requisito es que la costumbre haya sico observada pacíficamente y sin ser impuesta por la fuerza; el hecbo de ser disputada, o la amenaza de violencia física o moral para lograr su ojecución, serían prueba manifiesta de la carencia del consentimiento co. mún. El cuarto requisito es que la costumbre tenga fuerza obligatoria, que sea imperativa y no meramente facultativa, ya que de otro modo no si distinguiría de los usos sociales. En quinto lugar es necesario que la costumbre no sea incierta, pues no puede haber conciencia de la obligatoriedad de algo que no se está seguro qué es. El sexto requisito es que la costumbre sea congruente con las demás costumbres, ya que no puede haber dos cosiumbres inmemoriales $\mathrm{y}$ opuestas. Y el último requisito, $\mathrm{Y}$ el mós difícil de establecer, es que la costumbre debe ser razonable. El criterio que si-

(19) Dehnis LLOYD. Op. cit. p. 340. Sobre la relación entre usos de comercio y la ley. ver Patrick DEVUN. Samples of Lawmaking. London, 1962 p. 28.

(20) Sir Paul VINOGRADOFF. Op. cit. p. 121.

(21) Que tué el primero del reinado de Ricardo I. Sir Carleton K. ALLEN. Op. cit. p. 130. 
guen los tribunales para establecer to que es razonable es, generalmente, "lo que es justo o apropiado" $y^{\prime}$ "lo que haría una personx honesta e imparcial" (22); bien entendido que la calidad de razonable debe juzgarse no de acuerdo al criterio actual, sino al que se supone que haya prevalecido al tiempo de originarse la costumbre.

Las decisiones de los tribunales constituyen la fuente de Derecho más significativa y característica del Derecho inglés (23). Su aplicación se inicia después de la llegada a Inglaterra de Guillermo el Conquistador en 1066. En las postrimerías del siglo XIII, la práctica de citar fallos anteriores, como fundamento de un derecho, ya era frecuente en los estrados judiciales; y los autores recomendaban que al plantearse lin caso semejante $\alpha$ otro anterior se debía aprovechar la oportunidad para dar una solución análoga. En lc primera mitad del siglo XIV la citación de precedentes era tón común que se comenzó a considerar como una parte integrante de los alegatos, aunque todavía la mayoría eran mencionados por el propio tribunal, más que por los litigantes. En el siglo XV los jueces tomaron una conciencia más clara sobre la cplicación de los precedentes y al expedir fallos sobre materias no juzgadas antes, algunos declaraban expresamento su intención de establecer un precedente. En esta etapa el sistema inglés comenzó a elaborar una técnica judicial que era exactamente opuesta a la de Justiniano - que exigía fidelidad al principio general de la ley - y que se convirtió en uno de los principios rectores del llamado "Common Law", "Case Law" o según la expresión de Bentham, "judge made Law" (24). En el siglo XVI el abultado número de precedentes que se habían ido acumulanda creó la necesidad de las primeras recopilaciones de fallos, que al comienzo sólo mencionaban el nombre de las partes y la fecha del fallo, y que evolucionaron hasta convertirse, siglos más tarde, en los actuales "Law Reports", instrumento de trabajo indispensable para jueces, abogudos Y estudiantes. El siglo XVII vió crecer la autoridad de los precedentes judiciales hasta el punto de hacer declarar a un tribunal que - en vista de ausencia de fallos anteriores - gozaba de absoluta libertad para sen. tenciar. En el siglo XVIII Lord Mansfield Y sus discípulos observaron los peligros que acarreabx el uso mecánico y poco inteligente de los precedentes, y $\in$ l olvido de los principios y normas que estos establecían. Quedó en esta época establecido en doctrina, que son la razón y el espíritu de los

(22) En ingiés "fair and proper", y "such as reasonable, honest and fair minded men would adop!", respectivamente, Cit. por Sir Carleton K. ALLEN. Op. cit. p. 136.

(23) Sir Paul VINOGRAdOFf. Op. cit. p. 123. Sir Carleton K. ALLEN. Op. cit. p. 157. William GELDART. Elements of English Law Oxford. 1953. p. 7. Dennis LLOYD. Op. cit. p. 386 .

(24) La expresión "Common Law", se emplea dentro de tres contextos diforentes: a) para indicar el Derecho unificado de Inglaterra y Gales, on oposición a las costumbres de los condados; b) para indicar el Derecho elaborado por los jueces $y$ tribunales; c) para distinguir el Derecho de los paises que siguen el sistema legal inglés, en oposición a los de tradición civil y romanista. 
precedentes, y no las palabras o texto de estos, los que crean el Derecho. En el siglo XIX, se asentaron las bases de la doctrina moderna que está contenida en dos principios: el "stare decisis" y "la ratio decidendi".

Por el primero, un tribunal queda obligado por sus propios fallos y por los de los tribunales de jerarquía superior a la suya. En Inglaterra el más aito tribunal es la Cámara de los Lores $o$, más específicamente un comite judicial de esa Cámara; luego vienen la Corte de Apelaciones y la Corte de Apelaciones Criminales; y luego la Alta Corte con sus tres divisio. nes: la Banca de la Reina; la Cancillería; y la división de Testamentos. Divorcios y Almirantazgo, y el Tribunal Criminal Central. Todos estos tribunales juntos constituyen lo que se conoce como la Suprema Corte de la Judicatura, y son los únicos cuyos fallos pueden ser abligatorios. Otros triburales de jerarquía inferior como los de los condados, o los juzgados de paz no obligan con sus fallos sino a las partes del juicio (25). Sin embargo, cualquiera que sea el tribunal de origen, toda sentencia pertinente es un argumento sólido que merece consideración.

La "ratio decidendi" de un caso es en, términos generales, la razón. - espíritu del fallo, el principio en que está basado, y su única parte obligatoria para el tribunal que lo pronuncia y los de inferior jerarquía. Encontrar la "ratio decidendi" de un caso es, pues, de primordial imporiancia y constituye no pocas veces una tarea laboriosa. El primer paso que se debe dar es determinar todos los hechos del caso, tal como han sido vistos por el Juez; luego descubrir cuáles de esos hechos ha considerado el juez perti. nentes a la acción. Este segundo paso envuelve mayores dificuliades que el primero, pues muchos jueces proceden con cautela e incluyen hechos que no son esenciales para el fallo. El tercer paso consiste en relacionar los hechos declarados pertinentes con la norma o solución del caso (26). Pero una dificultad peculiar de los casos ingleses radica en que el fallo del tribunal es pronunciado por cada miembro en particular separadamente; $y$ el principio en que se basa el fallo puede ser concebido y expresado en términos diferentes por cada juez. En tales casos se debe buscar la "ratio decidendi" entre las opiniones de los jueces que están en mayoría. En un caso ya clásico, juzgado en el siglo pasado (27), quedaron estabiecidos los hechos siguientes: que el demandado habia hecho construir un depósito de agua en sus tierras; que el constructor había sido negligente;

(25) R. M. JACKSON. The Machinery of Justice in England. Cambriage. 1942. Caps'. II, III H, G. HANBURY. English Courts of Law. Oxford. 1953. Caps. V, VI, VII, Vlll. Peter ARCHER. The Queen's Courts. London, 1956 Secc. II. Las iniciales más usadas: Fara designar a los tribunales son: H. L. . Cámara de los Lores; C. A., Corte do Apelaciones: CH. D., Ia Cancillería: Q. B. D., la Banca de la Reina to K. B. D., Ia Banca del Rey); P.. Tribunal de Testamentos; D., Tribunal de Divorcios; y A., Tribunal del Almirantazgo.

(26) A. I. GOODHART The Ratio Decidendi of a Case. (1930). Yale Law Joumal. p. 161. Sir Paul VINOGRADOFF. Op. cit. p. 129.

(27) Rylands v. Fletcher (1868) L. R. 3 H. I. 330. 
y que el agua al escaparse habia ocasionado perjuicios al demandante. Pero, de estos hechos establecidos, la Cámara de los Lores consideró pertinentes el primero y el tercero; es decir, que el demandado había hecho construir un depósito de agua en sus tierras, y que el agua al escaparse había ocasionado perjuicios al demandante; y en base a estos hechos declaró que el demandado era responsable del perjuicio causado al demandante. Âhora bien, todo lo que los jueces digan respecto al segundo hecho, que el constructor había sido negligente, no obliga a nadie, ya que ésie no es parte en el juicic, ni los principios de doctrina que se declaren son aplicables a casos futuros, ya que se trata únicamente de opiniones dichas de paso y sin pe. sar los argumentos de las partes; esta parte del fallo se conoce como "obiter dictum" 0 , simplemente "dictum". Algunas veces también ocurre que el juez declara una norma mucho más amplia que los hechos considerados pertinentes requieren; en tales casos se tomará esía norma como "obiter dictum" y no como la verdadera "ratio decidendi" del caso. Aunque el tiempo no derogc a los precedentes judiciales sino que les da mayor fuerza, sin embargo si un precedente es muy antiguo se considera que no puede adaptarse a todas las circunstancias de la vida moderna, por lo que se le cita con menos frezuencia que los modernos. El texto de los fallos se encuentra en las recopilaciones, hechas por particulares, conocidas como "Law Reports". y de los cuales no se hace versión oficial (28).

La "Equity" es una fuente de Derecho peculiar y exclusiva del Derecho inglés y de los que se han inspirado en él (29). Se presenta habitualmente bajo dos formas: la interpretación liberal y humana del Derecho en general, o "Equity" general, y la modificación del Derecho, en casos excepcicnales que no caen dentro del ámbito de la normal general, o "Equity" particular. Ess, pues, la corrección del Derecho en lo que éste tiene de defectuoso debido a su universalidad; ejerce una influencia humana $y$ moderaciora sobre el Derecho. Pero, aunque tiene una relación íntima $y$ constante con éste, se originó y evolucionó separadamente, y constituye un cuer-

(28) Airque estas recopilaciones no son oficiales, sino que son tomadas en versión taquigráfica durante las audiencias, algunas son revisadas $\mathrm{y}$ hasta retocadas por los jueces y. naturalmente, gozon de mayor prestigio que las otras. Las más importantes son las cel "Incorporated Council of Law Reporting", que se conocen por las iniciales L. R. seguidas por las del tribunal respectivo, $Y$ una versión semanal del mismo (W. L. R. i; el "All England Law Reports" (AI E. R.); el "Times Law Reports" (T. L. R.); el "Law Times Reports" (L. T. R.). El fallo citado en la nota (28) p. ej. fue publicado por el "Incorpcrated Council of Law Reporting", en $l_{a}$ página 330, del tomo 3, de los tallos pronunciados en 1868 por la Cómara de los Lores.

(29) Este término ofrece un buen ejemplo de los problemas semánticos mencionados al comienzo de este trabajo. Ninguna de las traducciones que dan los diccionarios se aproxima lo suliciente. El "Dictionary of Legal Terms" de Robb da como equivalentes: equidad, derecho Iato, derecho equidad: Cuyás da: equidad, justicia: Cassell traduce: equidad, justicia, rectitud; Holt; equidad, y Velásquez; equidad, justicia, rectitud, imfarcialidad; téminos, todes, vagos que contrastan con la precisión definida de ia "Equity". Ver también G. R. RUDD. Op. cit. p. 25. Wiliam GELDART. Op. cit p. 21. Sir Paul VINOGRADOFF. Op. cit. p. 150. 
po de normas que, hasta el siglo pasado, eran aplicadas por tribunales especiales. In rigor, las reglas de "Equity" son, como las definió Maitland, una especie de suplemento 0 apéndice a las decisiones de los tribunales, pero muchas veces entran en conflicto con estas, ya que son también derecho judicial pero elaborado por tribunales distintos.

Las persona $a_{3}$ que recurrían durante la Edad Media, a la Corte cel Rey en demanda de justiria, algunas veces no veían satisfecho su deseo. ya fuese debido a la rigidez o a la deficiencia de la norma del Derecho. En casos tales, sólo quedaba al solicitante recurrir a la jurisdicción de "Equity" que la ejercía el Canciller - y de cionde se originó la Corie da la Cancillería actual --, o recurrir nievamente al hey con un recurso " $\alpha$ ? misericordiam". en que el soberano podía remediar las injus'ivias a quo dxba lugar la rorma. Es esta la facultad que Shakespecre destaca en boca de Porcia, en "El Mercader de Venecia", cuando dice que "la clemencia está por encima de la autoridad del cetro; tiene su trono en los corazone: de los reyes; es un atributo de Dios mismo, y el poder terrestre se aproxima tanio como es posible al poder de Dios cuando la slemencia atempera la justicia". Un caso ocurrido en el siglo XIV puede servir para ilustrar bien este punto (30). Un hombre se habia obligado a devolver un documento en una fecha determincta, y a pagar unce cantidad de dinero si no cumpía lo prometido. Demandado para pagar esta suma, se excusó diciendo qus no había cumplido por hallarse en olta mar, pero que habia dejado el $\mathrm{d}$. cunento on poder de su esposa y que hacía entrega de él ante el tribunal. Habiéndose acreditado los hechos expuestos, el juez, aunque por las nor. mas establecidas en los precedentes judiciales estaba obligado a declarar furcáada la demanda, expresó que, por "Equity", se demoraría siete años en expedir sentencia, obligando de este modo a transigir al demandante. Muchas veces, claro está, las soluciones de "Equity" distaban de ser justas, pero gradualmente se fué creando una jurisdicción apartə, para asuntes que tratasen del cumplimiento de obligaciones moraies, fraude, hechos ccurricos por accidente, anulabilided de contratos, interpretación de la intención de las partes de un contrato, protección de menores, incapaciáad do la mujer casada, y otros casos no contemplados por la ley. y cuya solución debía buscarse en la conciencia, o "conscience" que era otro de lns nombres con que se conocía a la "Equity". Enire las normas a instituciones a çue àió origen como fuente de Derecho, se encuentran las normas de propiedad independiente de la mujer casada (31), las compañíss de responsabiliciad limitada, y, su mayor creación, el "trust". Desdo 1875 la jurisdicción ordinaria y la de "Equity" estón unificades en los tribunales comunes, aunque las normas están hasia cierto punto separadas.

A cambio de las veniajas de certeza, carácter práctico, riqueza do normas detalladas y posibilidad de continuo desarrollo, el derecho jurisprudencial ofrece, sin embargo, las desventajas de rigidez, complejidad $Y$

(30) Cit. por Sir Carleton K. AlLEN. Op. cit. p. 385.

(31) Ver a este respecto del autor, la Familia en el Derecho Inglés. Boletín del Instituto do Derecho C'smparado de México. 1962. N N5 $^{\circ}$ p. 601. 
el peligro de crear distinciones ilógicas. Algunos de estos inconvenientes se remedian con la legislación, o "Statuie Law" que es la fuente de Derecho de mayor jerarquía en Inglaterra. Según la definición clásicc, parci ser ceconocida como tal, desde el siglo XVIII la legislación debe haber recibido la triple aprobación del Rey, los Lores y los Comunes. Antes de esa fecha no parece haber estado firmemente establecida la obligatoriedad absolutx e ilimitada de la ley (32). Il Derecho canónico, la "Eryity", y la intervetación de los jueces, interferian con frecuencia en los dispositivos de la ley, quizás debido en prirte $\alpha$ que esta era considerada más como guía y norma de gobierno que como meliculosa instrucción para probiemas prácticos. Desde Bentham hasta nuestro tiempo la importancia de la legislación ha ido en constante aumento. Después de la reforma del sistema electoral en 1831 el habitanie medio ha tomado conciencia de su posibilidad de influir en la legislación, a través de las numerosas organizaciones que canalizan la opinión pública, en las que quizás mejor que en ninguna otra actividad se ha revelado el genio peculiar del pueblo inglés (33). Aunque en Inglaterra la legislación no ha adoplado la forma de codificaciones ganerales, de tradición romanista, existen leyes promulgadas por el Porlamento en casi todas las ramas del Derecho; pero en donde esta fuente ha hecho sentir su presencia de modo más conspicuo y necesario ha sido en el Derecho público, y mós notablemente en el Derecho Administrativo después de la guerra, y en la legislación de seguridad social del llamado Estado benefactor. Otro especto importante ha sido que la revisión y modificación del Derecho puede lograrse más eficazmente por medio de la legislación, que no tiene el treno del principio del "stare decisis".

Las leyes, o "Acts of Parliament", pueden ser citadas de dos maneras: por el nombre oficial, generalmente consignado en un dispositivo de cada ley, que incluye la fecha de promulgación; o por el año de reina. do del monarca que promulgó la ley, y el capítulo del libro respectivo (34). La complejidad y la gran demanda de materias que requieren ser resueltas por la legislación ha dado lugar al desarrollo de lo que se conoce ccmo legislación delegada, por la que el Parlamento delega sus funciones legislativas, en determinado asunto, a un departamento gubernamental (35). Este tipo de legislación se da bajo la forma de "Order", o "Order in Council". "Regulation", "Rule" y "Schedule". Los "Statules" ○ "Acts of Parlia-

(32) Sir Carleton K. ALIEN. Op. cit. p. 431.

(33) A. V. DICEY. Op. cit. G. D. H. COLE., Raymond POSTGATE. The Common People. London, 1949. Graham WALl.AS. Human Nature in Politics. London, 1919. p. 199. Harold LASKI. A. Grammar of Politics. London, 1941. Ver dos ejemplos recientez on los trabajos del autor. El Derecho Internacional Privado en Inglaterra; en Revista de Jurisprudencia Peruana. 1962. No 224. p. 1080; y La Familia en el Derecho Inglés, cit. p. 598.

(34) Así, la ley de delitos contra el patrimonio, es conocida y citada, como "The Larcency Act, 1916", o también como "6 \& 7 geo. 5, c. 50", es decir, la ley del capítulo 50. del sexto y sétimo años del reinado de Jorge $V$.

(35) Kenneth MACKENZIE. The English Parliament. London, 1951. p. 167. Sir Courtenay ILBERT. Parliament. Oxford, 1950. p. 74. 
ment" están formados por "Sections". "Subsections" y "Paragraphs"; y los otros dispositivos por "Árlicles" "Regulations" o "Rules" según el caso, y "Paragraphs" y "Subparagraphs".

Hay varios métodos de interpretación de las leyes, que han sido acogidos por los tribunales y la doctrina ingleses (36). Algunas veces el mismo Parlamenio prescribe la forma en que ciertas palabras o expresiones deben ser entendidas. La ley de Interpretación de 1899 (37), reunió las disposiciones dispersas que existían al respecto fijando normas como las que determinan que las palabras masculinas comprenderán las femeninas, los singulares comprenderán las plurales, y viceversa; que la palabra "persona" comprende cualquiera agrupación con personería jurídica o sin ella; o que la palabra "escritura" comprende impresos, fotografías, o cualquiera otra forma de reproducir palabras en forma visible. En la actualidad es bastante común que las leyes contengan una clóusula dedicada a definir el sentido de las palabras que emplean. El método más generalizado ha sido el de la interpretación literal. Según este método las palabras de una ley deben ser interpretadas de acuerdo a su significado literal, y las oraciones de acuerdo a su significado gramatical. Hay que dilucidar la "ratio verbcrum". Las palabras comunes $u$ ordinarias deben ser interpretadas de acuerdo a su significado ordinario, natural o popular; las palabras técnicas de acuerdo a su sentido técnico, $y$ en todo caso el significado de una palabra es el que tenía al tiempo de la aprobación de la ley. En la práctica judiciai han sido las palabras más comunes las que han dado origen a las mayores dificultades. Se debe considerar, ademós, que el significado de las palabras está condicionado por el contexto en que figuran, y que lo que se establece en una parte de una ley, puede ser modificado en otra (38). Los principales defectos de este método son, primero, precisamente que el significado de las palabras varia de acuerdo al contexto en que están; luego, que el concepto mismo de "interpretación" denota la introducción de elementos extraños a las palabras, por insuficiencia de estas; que ningún sistema se ha librado de las paradojas de la interpretación literal o gramatical; y que, en última instancia, la interpretación literal no es sino un medio para llegar al espíritu o intención de la ley. la "ratio legis" (39).

Los métodos restantes están dedicados a hallar la intención de la leY̌ o "ratio legis". El primero de ellos es conocido como "The Golden Rule", que en apariencia no es sino unj variante de la interpretación literal; pero que se diferencia de esta en que se aparta de la significación literal o gramatical cuando puede dar lugar a resultados absurdos, o a incongruencias con los demás dispositivos de la misma ley. Otro método de hallar el propósito general o espíritu de la ley es el llamado "Mischief Rule". y que

(36) Sir Carleton K. ALLEN. Op. cit p. 468. Sir Paul VINOGRADOFF. Op. cit. p. 88. O. Hoods PHILLIPS. Op, cit, p. 91 . A. K. R. KIRALFI. Op. cit. p. 117.

(37) The Interpretction Act, 1889

(38) O. Hoods PHILLIPS. Op. cit. p. 95.

(39) Sir Carleton K. AlLEN. Op. cit. p. 492. 
se aplica averiguando lo que establecía el Derecho antes de la promulgación de la ley, cuál era la falta o conducta que la ley no proveía, cuál el remedio o solución que propuso el Parlamento; y cuál la verdadera $\mathrm{r}$ בzón de la solución final (40). Además, se puede recurrir al auxilio de varios elementos internos de la ley, como el título con que se le cita, la introducción o preámbulo, si lo tiene, la puntuación, los encabezamientos, legisiación delegada, reglamentos, y las notas marginales a la misma ley. Está expresamente excluído el uso de trabajos preparatorios, actas y discusio. nes de las comisiones, y debates parlamentarios, y no se puede citar en apoyo de una interpretación la intención del legislador. Es cierto que el uso indiscriminado de estos elementos puede dar origen a debates interminables, pero su supresión radical es una medida extrema que fue satirizazada desde los tiempos de Jonathan Swift, en uno de cuyos libros de viujes imaginarios se cuenta de un país en el que escribir comentarios sobre lae leyes era un delito que se castigaba con la pena capital.

Ademós de las tendencias mencionadas al principio de este træbajo. el pensamiento jurídico inglés se ha desarrollado principalmente dentro de tres cauces. El más antiguo es el Derecho Natural (41), que se incorporó a través del Derecho canónico y de obras como "Doctor and Student" de Saint Germain, en que su autor trazó una comparación entre el Derecho canónico y el Derecho inglés del siglo XVI. William Tyndale fue uno də los primeros pensadores en acoger esta doctrina y declarar que el Derecho es la ley de la Naturaleza y de la equidad natural que Dios ha puesto en el corazón de los hombres. Con la Reforma Protestante, que identificó el Derecho Natural con el catolicismo, y con el surgimiento de los prime:os juristas profesionales, más interesados en el aspecto positivo de las leyes que en su estructura filosófica, la doctrina se eclipsó temporalmente. Uno de esto:s juristas, Sir Edward Cocke, lector y admirador de Saint Germain, hizo un primer intento de retorno, al delender el punto de vista que una ley promulgada por el Parlamento puede ser nula, si va contra la justicia. Coke gozaba de influencia suficiente como jurista, para poner en primer plano el problema de determinar la existencia de una ley que tuviera mayor autoridad que los mandatos del rey. Los católicos volvieron a apoyar sus reclamos amparados en el Derecho Natural que proclamaba la igualdad de los hombres; esta vez, sin embargo, su voz no fué la única, ya que los puritanos no tardaron en valerse del mismo argumento, manifestándose alguinos de los mayores defectos de esta doctrina que podía citarse en apoyo de afirmaciones dispares, y aún contradictorias.

Fué debido a Richard Hooker y a su doctrina, inspirada en Santo Tomás y en la teoría de la independencia entre el Estado y la Iglesia de Marsillo de Padua, a quien se debe la aceptación del Derecho Natural como compatible con la teología protestante. Hooker tomó de Santo Tomás la idea de que si una: ley positiva es contraria al Derecho Natural o al Derecho

(40) O. Hoods PHILLIPS. Op. cit. p. 97.

(41) Dennis LLOYD. Op. cit, 53 y siguientes; Christopher MORRIS Op. cit. p. 128 y siguientes. 
Divino puede ser desobedecida; y de Marsillo de Padua la afirmación de que el Derecho positivo debe fundarse en el consentimiento común. De Hooker la doctrina pasó a John Locke quien ha sido su más brillante expositor en Inglaterra (42). Reconoce Locke la existencia de ciertos derechos nchurales que identifica con la vida, la libertad y la propiedad. Pero en el estado de naturaleza falta la autoridad que garantice los derechos del individio, y para suplir esta deficiencia es que debe renunciar $\propto$ parte de su libertad a favor de un poder central. Este poder, sin embargo, no puede gozar de mayores privilegios que los que se desprenden de la suma de voluritades individuales que le dieron creación, y por consiguiente no puede ir contra los dictados de la ley natural. De este modo, Locke empalma la dostrina del Derecho Natural con la del Contrato Social. En el siglo XVIII Sir Wiliam Blackstone intentó, con relativo éxito y por un corto periodo reavivar la doctrina volviendo a la división tomista del Derecho en Divino, Natural y Positivo. En la actualidad el Derecho Natural es invocado muy rara vez, y generalmente bajo la forma del orden público, los derechos humanos o la justicia natural.

La segunća corriente filosófica, es la conocida con el nombre de teoría imperativa o de soberanía del Estado. Ya hemos mencionxdo, líneas más arriba, el punto de vista de esta teoría en relación a las fuentes de Derecho. Thomas Hobbes, uno de los primeros juristas identificado con esta doctrina, pertenece a la clase de pensadores, aparecida durante el Renacimiento, que tratan de afirmar la autoridad absoluta del Estado frente a las demás influencios, y que está representada en Italia por Niccolo Macchiavelli, y en Francia por Jean Bodin. Hobbes, como años mós tarde haría Locke, recogió la doctrina del contrato social y le dió un contenido personal. El Estado de la naturaleza es un estado perenne de guerra, se supera sólo cuando se acuerda que un grupo de personas, o una sola persona, represente la voluntad de los demás durante un tiempo limiiado - para siempre (43). Si se acepta excepciones al deber de obediencia se deja una puerta abierta a la confusión y al caos. En el "Leviatán", su obra capital, deciara que el Estado es una creación artificial y omnipotente, que tiene por finalicad la protección del hombre, y que ningún ciudadano goza de derechos frente al poder público; la soberanía es una e indivisible, pues la división de poderes conduce a la disolutión del Estado.

Aunque esta doctrina pasó al olvido después de la muerte de Hobbes, la confianza en los poderes del gobierno para satisfưcer las necesidades de la sociedad sin recurrir al Derecho Natural, fué compartida por el Vizconde Halifax, Edmund Burke y Jeremy Bentham. Pora este último el gobieino era piedra angular para iniciar las reformas constitucionales, legales, sociales y económicas por las que abogaba, tales como el sufragio universal, el voto secreto, el gobierno representativo, la reforma carceloria y la legislación codificada para lograr mejor estos fines (44). Pero no fué

(42) Jchn LOCKE. Op. cit.

(43) G. P. GOOCH. Op. eit. p. 29.

(41) Jeremy BENTHAM, The Theory of Legislation, London, 1950. p. 13 
Bentham sino su discípulo John Auptin, inspirado en los esfuerzos de Ins juristas alemanes de la Escuela Histórica para elaborar una ciencia del Derecho, quien revivió la doctrina de Hobbes para con ella elaborar una teoría general del Derecho (45). Para Austin el objeto propio de la jurisprudencia es el Derecho positivo, es decir, el Derecho estableciỏo en una ccmunidad política independiente por la voluntad expresa o tácita de su soberano o gobierno supremo. Es uno de los primeros en declarar que "hay principios, nociones $y$ distinciones comunes a varios sistemas, las cuales forman analogías o semejanzas que los hacen afines", y que la ciencia que estudia estos principios "ha sido llamada Jurisprudencia general. o comparada, o bien filosofía - o principios generales - del Derecho positivo" (46). Exses de esta teoría general son los conceptos de deber. derecho, libertad, delito, pena, resarcimiento, derecho escrito $Y$ no escrito, derechos absolutos y relativos, derecho objetivo y subjetivo; pues la Jurisprudencia es la ciencia de lo que es esencial al Derecho, así como la ciencia de lo que el Derecho debe ser. Enire los más destacados continuadores de esta escuela se cuentan T. E. Holland, F. Salmond, J. Gray y F. Pollock.

Ia tercera tendencia del pensamiento juridico inglés está constituida por la Escuela Histórica de Juriepruóencia representada por Sir Henry Maine y F. W. Maitland. El pensamiento de Maine sigue, por una parte, la huella trazada por Montesquiau en "El Espiritu de las Leyes" para destacar que tcdo Derecho es el producto de una época y lugar determinados $y$, de otra. parte, la doctuina de la Escuela Hisiórica alemana, tal como fué expuesta por ihering, para reveiar la importancia normativa de la costumbre comn tucnte de Derecho. Su cbra más importante estuvo decicada al estudio del Derecho en los pueblos primitivos y las civilizaciones mós antiguas, y $a$ llamar la atención sobre la ventaja y provecho que pueden seguir del estudio del Derecho Romano (47). Varios capítulos de su obra fueron dedicados a combatir las exageraciones en que habian incurrido los racionalistas al describir el estado de naturaleza y el contrato social. El método propuesto por Maine fué, histórico y comparativo a la vez como acertada. mente ha observado Sir Ernest Baker (48). Como Montesquieu en Francia, Maine fué en Inglaterra el iniciador del estudio comparado del Derecho. De lx afirmación que todo Derecho es el producto de una realidad consreta, pasa el estudio de otros derechos y de su función en distintas realidades. Un brote de esta escuela ha sido el moderno método comparativo, expuesto con brillantez difícil de igualar por H. C. Gutteridge (49), quien señalu como las metas de éste el mayor acercamiento de los pueblos mediante el conocimiento de su Derecho y sus leyes; y cuyos más destacados representantes en la actualidad son C. J. Hamsom, Lord Mc Nair, K. Lipstein y F. H. Lawson.

(45) R. A. EASTWOOD. Introduction to Austin's Theory of Positive Law and Sovereignty. London, 1918. p. 15.

(46) John AUSTIN. Op. cit. Hay un $a$ traducción al español, recomendable, de algunos copífulos, por Felipe González Vicen Madrid, 1951.

(47) Op. cit.

(48) Sir Emest BAKER. Op. cit. p. 143.

(49) H. C. GUTTERIDGE. Comparative Law Cambridge, 1946, p. 5. 